\title{
COVID-19 Pregnancy and Fertility: A Narrative Review
}

\author{
Tiffany Field
}

Department of Pediatrics, University /Miller School of Medicine, and Fielding Graduate University, USA

Corresponding Author: Tiffany Field, Department of Pediatrics, University of Miami/Miller School of Medicine.

Received date: September 13, 2021; Accepted date: September 17, 2021; Published date: October 18, 2021

Citation: Field T. 2021) COVID-19 Pregnancy and Fertility: A Narrative Review. J. Obstetrics Gynecology and Reproductive Sciences 5(8); DOI: $10.31579 / 2578-8965 / 097$

Copyright: ( ) 2021, Tiffany Field, This is an open access article distributed under the Creative Commons Attribution License, which permits unrestricted use, distribution, and reproduction in any medium, provided the original work is properly cited.

\begin{abstract}
Pregnant women are currently worrying about both the effects of COVID-19 infection as well as the vaccine on their fetus and newborn. This narrative review is a summary of the literature on COVID-19 psychological problems of pregnant women, and infection effects on the women, their fetuses and neonates. The psychological problems studies have consistently shown a prevalence of approximately $20-40 \%$ anxiety and depression in pregnant women during COVID-19. Although early pandemic research suggested that the severity of COVID infection and the clinical course for infected pregnant women was no worse than for non-pregnant infected women, later pandemic, larger sample studies and meta-analyses suggest that infected pregnant women have more obstetric complications and negative outcomes than pregnant women without infection. Greater prevalence of pre-eclampsia, fetal distress, premature rupture of the membranes and preterm delivery have been reported for pregnant women who are infected, especially during the third trimester. Older pregnant women and those with comorbidities including elevated BMI, diabetes and hypertension are also at greater risk for obstetric complications. Mixed data have been presented on vertical transmission of the virus to the fetus as well as questions about vertical transmission via reduced natural killer cells protecting the placenta and increased ACE-2 receptors. Although the neonatal infection rate has been relatively low, antibodies noted in some neonates ( $\operatorname{IgG}$ and $\operatorname{IgM}$ ) suggest intrauterine, delivery or postnatal transmission. The very limited data on vaccination during pregnancy suggest positive effects. Although the data are even more limited on vaccination and fertility, there are no known negative effects. Methodological limitations of this literature include the data having been cross-sectional and derived from samples of symptomatic, hospitalized pregnant women at different gestational ages and different times during the pandemic.
\end{abstract}

Keywords:covid-19;pregnancy; fertility; fetus; newborn

\section{Introduction}

The literature on COVID-19 effects on pregnancy, the fetus and the neonate is extensive. Hundreds of studies and review papers published in 2020-2021 have reported on these effects. The present review of the literature is a non-technical summary of studies and review papers on COVID effects on the pregnant woman, the fetus and the neonate as well as the assessment of vaccine effects on pregnancy and fertility. The terms COVID-19, pregnancy, fertility and vaccination were entered into the PubMed advanced search engine. That literature search yielded 136 papers including systematic and narrative reviews of the literature. Following exclusion criteria of case studies and foreign language papers and the inclusion of peer-reviewed papers, this narrative review includes brief summaries of the findings from 34 publications including 14 systematic and narrative review papers. This paper is organized around the topics that are reflected in that literature including effects on the pregnant woman, the placenta, the fetus, and the newborn as well as vaccine effects on pregnancy and fertility and methodological limitations of the literature.
To provide a brief synopsis, two separate literatures have emerged, with one addressing the psychological problems of pregnant women during COVID-19 and the other assessing the effects of infection on pregnant women, their fetuses and newborns. The psychological problems literature consistently shows a prevalence of approximately $20-40 \%$ depression and anxiety in pregnant women during COVID-19. But this literature doesn't address problems of infection. And the infection research has not assessed the potentially confounding effects of depression and anxiety on COVID-19 infection in pregnant women. The early literature on infection effects generally suggested that pregnant women with COVID were not notably different from non-pregnant women with COVID in terms of the severity of infection and their clinical course. However, the effects of COVID infection are confounded with pregnancy complications that don't necessarily derive from infection, making comparisons between pregnant and non-pregnant women less valid. Larger sample studies and meta-analyses on comparisons between pregnant women with and without COVID infection in the more recent literature have suggested more frequent obstetric complications and negative outcomes for pregnant women experiencing COVID infection 
versus pregnant women who are not infected. Those complications include a greater prevalence of pre-eclampsia, fetal distress, premature rupture of the membranes and preterm birth. Although the evidence is mixed for vertical transmission of the pregnant woman's infection to the fetus, COVID infection has been detected in amniotic fluid. In addition, despite relatively limited infection noted in neonates, evidence of IgM and IgG antibodies in the newborn suggests fetal development of those antibodies and vertical transmission inasmuch as those antibodies are not known to cross the placenta. Although the placenta is thought to be an effective barrier that prevents maternal infection spreading to the fetus, given that it is surrounded by natural killer cells, the potential for viral invasion of the placenta is not clear. And, there are mixed data on ACE-2 (angiotensin converting enzyme) receptors (that receive the virus) in the placenta.

In addition, concerns have been raised about vaccine effects on pregnancy and fertility. Although there is limited research on those questions because pregnant women were not included in the vaccine trials, there are at least two studies indicating the absence of vaccine effects on fertility.

\section{COVID-19 Effects on Pregnant Women \\ Psychological Problems}

With regard to the psychological problems literature, a few metaanalyses have highlighted the prevalence of anxiety and depression in pregnant women during COVID-19, but infection was not assessed. In one meta- analysis on 11,187 pregnancies from 15 studies, depression was noted in $30 \%$ of the women, anxiety in $34 \%$ and comorbid depression and anxiety in $18 \%$ (Sun et al, 2021). The authors reported that all these problems occurred more frequently in pregnant women than in the nonpregnant women controls. They also noted extreme heterogeneity of the data.

In another meta-analysis on 19 articles, $25 \%$ were depressed, $42 \%$ were experiencing anxiety and the younger pregnant women experienced greater anxiety (Fan et al, 2020). This meta-analysis included studies from China, Israel, Italy, Belgium and Turkey. After depression and anxiety, fear and stress were the most commonly experienced psychological problems. Again no mention was made of infection and again, the authors highlighted the fact that the data were highly heterogeneous.

In a third meta-analysis on 46 studies, depression was noted in 37 studies $(\mathrm{N}=47,677)$ at a prevalence of $26 \%$ and anxiety was noted in 34 studies $(\mathrm{N}=42,773)$ at a prevalence of $31 \%$ (Tomfohr-Madsen et al, 2021). The authors reported that anxiety was more prevalent in studies conducted later in the pandemic. A few studies that have appeared more recently include one on- line survey that included the Pregnancy-Related Anxiety Scale on pregnant women ( $=2740)$ from 47 states (Moyer et al, 2020). 93\% reported increased stress about getting infected. The more COVID-related stressors the pregnant women experienced, the greater their anxiety levels.

In a cross-sectional survey during the first month of lockdown in Italy, no women reported infection ( $\mathrm{N}=737)$ (Ravaldi et al, 2021). Anxiety was noted in $22 \%$ of the women and PTSD in $10 \%$. Previous anxiety and depression were independently associated with PTSD. Previous anxiety was a greater predictor of PTSD in those who experienced greater worries about the health of the baby. In another cross-sectional study $(\mathrm{N}=283)$, COVID-related symptoms predicted PTSD symptoms, although none of the women were diagnosed with COVID infection (Hocaoglu et al, 2020).

These studies collectively suggest that about a third of pregnant women during COVID-19 are experiencing worries about infection, anxiety, depression and PTSD. Despite the prevalence of these problems and their potential for confounding and compounding infection effects, they have not appeared in the literature on infected pregnant women. The lack of consideration of infection in the psychological studies may relate to: 1) the focus being exclusively on psychological problems in the larger population of pregnant women; 2) the studies being conducted early in the pandemic when the prevalence of infection was low; and 3) the research being based on survey and prenatal clinic samples as opposed to hospital records that were the primary data base for the infection studies.

\section{Infection Effects}

Regarding the literature on infected pregnant women, small sample studies at the beginning of the COVID-19 pandemic suggested that pregnant women did not differ from non-pregnant women on the severity of their COVID infection and symptoms (Maleki et al, 2020). The prevalence of different level severity among COVID-infected pregnant women appeared to be similar to that of non-pregnant women including $80 \%$ mild, $15 \%$ severe and 5\% critical (Ryan et al, 2020). Like nonpregnant women, the COVID symptoms for pregnant women were fever (62\%) and cough (45\%) (Mirbeyk et al, 2021). However, a CDC report of June 2020 suggested that $32 \%$ of pregnant women with COVID needed hospitalization versus $6 \%$ of non-pregnant women (Elsaddig \& Khalil, 2021). The odds were 1.62 greater for pregnant women with COVID to require hospitalization and $1.88 \%$ more likely to require invasive ventilation than non-pregnant women with COVID (Elsaddig \& Khalil, 2021). In another study, $11 \%$ of women with COVID required intensive care (Elsaddig \& Khalil, 2021). Most frequently noted indications for hospitalization were pneumonia, acute respiratory disease syndrome, pulmonary embolus and acute coronary syndrome (Elsaddig \& Khalil, 2021).

As has been suggested, a more relevant assessment of the additive effects of COVID on pregnancy complications would be comparisons between pregnant COVID-infected versus pregnant non-infected women given that changes naturally occur during pregnancy (Wastnedger et al, 2021). Typical pregnancy changes include, for example, enhanced immune function, cardio-pulmonary changes and increased demand for oxygen for the mother and the fetus (Kumar et al, 2021). When pregnant women with and without COVID have been compared, more obstetric complications have been noted for those with COVID. For example, recent large sample studies and meta-analyses have suggested significantly more obstetric complications in pregnant women with COVID versus those without COVID. In a review of 20 studies including 230 pregnant women, for example, 35\% more pregnant women with COVID were reported to have obstetric complications (Chi et al, 2020).

Obstetric complications were noted primarily in the third trimester (88\%) in a systematic review of 37 papers based on 364 pregnant women (Mirbeyk et al, 2021). Others reported greater odds for the need of intensive care during the third trimester (Elsaddig \& Khalil, 2021). But these authors suggested that the greater need for intensive care may reflect a lower threshold for providing treatment for late pregnancy women with COVID rather than severity of their obstetric complications. However, that severity would be greater during the third trimester is not surprising given that it has been noted as a pro-inflammatory period (Narang et al, 2020). Reputedly, for example, there is a decrease of the protective antiviral, natural killer cells at this time as well as an upregulation of ACE-2 receptors (Narang et al, 2020). Others have also reviewed data suggesting that there is a suppression of natural killer cells and $\mathrm{T}$ cells late in pregnancy as well as a twofold increase in ACE-2 receptors in the placenta (Kumar et al, 2021).

Early studies suggested that unlike MERS and SARS, COVID did not affect the maternal death rate. However, one of these studies was based on a small sample $(\mathrm{N}=38)$ and occurred early in the pandemic (Schwartz, 2020). It was not clear why the similar MERS and SARS viruses could lead to a greater prevalence of maternal death than COVID-19. But, the 
death rate cited in the $2020 \mathrm{CDC}$ report was $70 \%$ greater for pregnant versus non-pregnant women with COVID and was attributed to acute respiratory distress syndrome related to significant inflammation (Wastnedger et al, 2021). However, again, the comparison between pregnant women with and without COVID may have been more relevant than the more frequent comparison between pregnant women with COVID versus non-pregnant women with COVID in order to determine the combined effects of pregnancy plus COVID. Further, the pregnancy status in the CDC report was only reported by $50 \%$ of the sample (Elsaddig \& Khalil, 2021). Notably, the pregnant women with COVID in that sample were more likely to be minority status including Hispanic or Black (Elsaddig \& Khalil, 2021). In addition, they were not only lower socioeconomic status, but they also had a greater incidence of comorbidities including vitamin D deficiency, diabetes, chronic hypertension and higher BMI levels (Elsaddig \& Khalil, 2021). Further, in another recent review, older pregnant women and those with preexisting comorbidities including hypertension, diabetes and cardio pulmonary disease were at a greater risk of mortality (Kumar et al, 2021).

\section{COVID-19 Obstetric Complications and Effects on the Fetus}

Several obstetric complications have been noted in the COVID-19 literature including pre-eclampsia, fetal distress, premature rupture of the membranes, Caesarean section and preterm delivery. Although as many as $27-35 \%$ of pregnant women with COVID are noted to have these pregnancy complications (Chi et al, 2020; Moore \& Suthar, 2021), the prevalence has been highly variable, likely as a function of the severity of the mother's infection and the source of information for the various studies and reviews which has mostly been medical records.

Miscarriage and stillbirths have been discussed in the context of insufficient data on prevalence (Elsaddig \& Khalil, 2021; Panahi et al, 2020). In contrast, sufficient data on termination of pregnancy have suggested that it has increased across the pandemic which has, in turn, been related to the fear of fetal infection (Elsaddig \& Khalil, 2021).

Pre-eclampsia has been noted by several investigators (DiMascio et al, 2020; Kumar et al, 2021; Narang et al, 2020; Wastnedger et al, 2021). The prevalence has varied from $16 \%$ noted in asymptomatic COVID-19 (DiMascio et al, 2020) to 63\% in the case of severe COVID-19 (Kumar et al, 2021). Some have attributed pre-eclampsia to the elevation of the cytokines including IL-6 and the associated increase in inflammation (Moore \& Suthar, 2021).

Fetal distress (insufficient oxygen through the placenta) has surprisingly been mentioned in only a few reviews. In one review, $14 \%$ of COVID-19 pregnancies involved fetal distress and 8\% ruptured membranes, (Kumar et al, 2021). In a narrative review of 13 studies, fetal distress and miscarriage rates were not different for COVID versus nonCOVID pregnancies (Panahi et al, 2020). However, in this review, those pregnancies also did not differ on prematurity rates which is inconsistent with data from most of the other reviews. For example, in a review of 21 studies, that only included 90 pregnant women, fetal distress was more frequent in pregnant women with COVID than pregnant women without COVID (Ashraf et al, 2020). The authors speculated that the fetal distress resulted from an overproduction of pro-inflammatory cytokines that they called a "cytokine storm". In a similar review based on 13 studies, the authors suggested a greater prevalence of fetal distress among pregnant women with COVID (Panahi et al, 2020). And in another review paper, as many as $14 \%$ of pregnant women with COVID had experienced fetal distress (Kumar et al, 2021). Not surprisingly, intra-amniotic infection can lead to an inflammatory cascade, in turn initiating spontaneous labor and preterm delivery, and intrauterine infection accounted for $40 \%$ of preterm deliveries in at least one review (Prochaska et al, 2020).
Caesarean section of course would explain a significant amount of the variance in preterm deliveries in COVID-infected women. The prevalence of Caesarean section has widely ranged from $43 \%$ to $92 \%$ in one review of the literature (Ryan et al, 2020). Those authors suggested that $\mathrm{C}$-sections were performed due to maternal respiratory problems. Another reason given for high Caesarean rates was excessive weight gain associated with less physical activity during the pandemic (Elsaddig \& Khalil, 2021). Others have variously reported a prevalence of $48 \%$ (Kumar et al, 2021), 57\% (Mark et al, 2021) and 84\% (DiMascio et al, 2020). The high $84 \%$ prevalence was based on a meta-analysis of 19 studies, but only 41 of the pregnant women were experiencing COVID. In a similar review on a high prevalence (86\%), $60 \%$ of the COVID infected pregnant women expressed fear of infection effects on their fetuses leading them to elective Caesarean sections (Mirbeyk et al, 2021). And $83 \%$ expressed concerns about infecting their newborn during delivery in another study (Akgor et al, 2021). This was especially prevalent among the older and more anxious pregnant women. Further evidence of elective Caesarean sections comes from a study from China in which the odds of a Caesarean delivery were 3.34 greater for women with COVID (Elsaddig \& Khalil, 2021). For these reasons, the greater prevalence of preterm delivery among COVID pregnant women has been called iatrogenic preterm delivery. Preterm delivery has been called iatrogenic reputedly because it derives from frequent Caesarean section deliveries related to maternal complications.

Independent of Caesarean sections, the prevalence of preterm delivery has been notably lower. For example, it has been noted as $13 \%$ in one study, which is not significantly different from $10 \%$ in the general population (Moore \& Suthar, 2021). The prevalence has been greater for symptomatic than asymptomatic women (19\% versus $9 \%)$. Others have reported prevalence of preterm births ranging from 20 to $30 \%$ including 22\% (Kumar et al, 2021), 25\% (Chi et al, 2020), 24\% (Mirbeyk et al, 2021), 28\% (Mark et al, 2021), suggesting some consistency across reviews of the literature, although an outlier of $41 \%$ has also been reported (DiMascio et al, 2020).

Significant cross-cultural differences have been noted for the prevalence of preterm delivery (Elsaddig \& Khalil, 2021). For example, the average prevalence of preterm delivery for women with COVID has been noted as $19 \%$ for Europe, $17 \%$ for China and $12 \%$ for the U.S. (Elsaddig \& Khalil, 2021). These rates seem inconsistent with the crosscultural differences in Caesarean section rates of 38\% in Europe, $91 \%$ in China and $40 \%$ in the U.S. (Elsaddig \& Khalil, 2021). These authors suggested that the wide variation in C-section prevalence is likely related to the differences in the stage of the pandemic and the relative thresholds for performing Caesarean sections. Nonetheless, iatrogenic preterm birth has been consistently more prevalent for COVID versus non-COVID pregnancies (Elsaddig \& Khalil, 2021).

The fact that $40 \%$ of cortisol is transmitted across the placenta (Glover, 2015) suggests that cortisol elevation could itself contribute to the prematurity that is being seen in the offspring of pregnant women experiencing COVID-19 infection. And, cortisol has notably explained a significant amount of the variance in preterm gestational age in a preCOVID study (Field et al, 2006). The transmission of cortisol across the placenta could have independent effects on the neonatal outcomes of women experiencing COVID-19 infection. The protective immunity of pregnant women may reduce the infection-related conditions and the vertical transmission of the infection to the fetus. Aside from some evidence of infection in amniotic fluid and the positivity in some neonates, it would appear that the immunity of pregnancy is not only protecting the pregnancy but also the fetus and the neonate. The greater prevalence of fetal distress and premature delivery may result from prenatal depression, elevated cortisol and the resulting reduction of natural killer cells independent of COVID-19 infection itself. The fetal 
distress and preterm delivery could be independently explained by the women's depression and anxiety and the related crossover of cortisol from the mother to the placenta causing premature rupture of the membranes and preterm delivery independent of infection or vertical transmission. However, there is increasing evidence for vertical transmission of COVID-19.

\section{Vertical Transmission of COVID-19}

Maternal-fetal immunity is a rapidly developing field (Prochaska et al, 2020). One of the continuing arguments in this literature relates to vertical transmission of the infection from the mother to the fetus. The placenta is thought to be an effective barrier to prevent maternal infection from spreading. The natural killer cells (at least $30 \%$ of protective cells) are thought to protect the placenta (Moore \& Suthar, 2021). In a review of 17 studies, the authors suggested that even $70 \%$ of the protective cells are natural killer cells that regulate the production of cytokines (especially IL-10) (Alberca et al, 2020). As the NK cells are decreased in COVIDinfected pregnant women, there is an increase of IL-10 as well as other pro-inflammatory cytokines including IL-6 and C-reactive protein (Alberca et al, 2020).

The absence of infection detected in most samples of newborns has led several to suggest the absence of vertical transmission (DiMascio et al, 2020; Maleki et al, 2020; Panahi et al, 2020; Schwartz 2020). However, data from larger samples have suggested infection in amniotic fluid, the presence of IgM in the umbilical cord and both IgM and IgG in newborns (Moore \& Suthar, 2021; Wastnedger et al, 2021). Because these antibodies reputedly cannot cross the placenta, in utero exposure has been assumed as well as development of those cells by the fetus. The same authors have suggested that while there may be a prevalence of $3-8 \%$ vertical transmission, this could also occur during delivery. In addition, they have acknowledged that the mechanism of viral invasion of the placenta is not clear. Further evidence for vertical transmission is that placentas from infected patients have shown inflammatory, thrombotic and vascular changes that have been found in other inflammatory conditions (Prochaska et al, 2020).

\section{Viral Invasion of the Placenta}

COVID infection has been noted to trigger hyper-activation of inflammatory and immune responses resulting in a "cytokine storm" and an increased inflammatory response in the placenta (IL-6, IL-8,IL-10, TNF) (Kumar et al, 2021). The protection of the placenta by surrounding natural killer (NK) cells may also be compromised by COVID infection (Narang et al, 2020).

Another contributor to inflammation, as already mentioned, is related to prenatal depression and anxiety that are notably exacerbated during COVID-19 for fear of infection (Wastnedger et al, 2021). That would predictably lead to elevated cortisol (which can cross the placenta) and its negative effects on immune function, most particularly its reduction of natural killer cells. Inasmuch as natural killer cells are a protective factor for the placenta, an infection could invade the placenta via a reduction of the protective natural killer cells (Narang et al, 2020). Natural killer cells surrounding the placenta could in part be depleted by their activity in warding off the viral cells infecting the pregnant woman. Given that there is a significant literature on the negative effects of prenatal depression and anxiety leading to increased cortisol levels and prematurity, it is noteworthy that this possibility has not been discussed in this literature on COVID-19 pregnancy (Field et al, 2006).

Further, as already mentioned, the data are mixed on the presence of ACE-2 receptors in the placenta, as was noted in a review of 31 studies including one of the largest samples in this literature $(\mathrm{N}=12,260)$ (Wastnedger et al, 2021).

\section{Newborn Course}

The more recent literature has documented evidence of the neonate having been infected. In a UK study, 12 of 265 infants were infected (Kumar et al, 2021). Of those neonates, $4 \%$ had G.I. problems, 3\% had fever and 6\% had shortness of breath (Kumar et al, 2021). In a CDC study $32 \%$ of infected neonates required hospitalization (Kumar et al, 2021). In a meta-analysis, $22 \%$ were preterm, $48 \%$ had Caesarean deliveries and $7 \%$ required intensive care (Kumar et al, 2021).

Based on a review of 60 studies totaling 1287 neonates, the authors concluded that it's still unclear whether neonatal COVID derived from infection in utero, intrapartum or post-partum (Pettirosso et al, 2020). The elevated levels of antibodies including $\operatorname{IgM}$ and $\operatorname{IgG}$ that have been detected in neonates of COVID-infected mothers, has suggested in utero exposure (Moore \& Suthar, 2021). In a review on 37 studies, high IgM levels were reported in at least two studies (Mirbeyk et al, 2021). In this review, 3 out of 8 newborns with negative throat swab tests had elevated IgM and IgG against SARS COV-2, suggesting a vertical transmission rate of $4 \%$ (Mirbeyk et al, 2021). These authors also argued that there is no possibility of IgM crossing the placenta. In addition, pro-inflammatory cytokines including IL-6 have been found in neonates of mothers who only had mild COVID (Moore \& Suthar, 2021).

The infection symptoms noted in neonates have included dyspnea $(42 \%)$ and fever (19\%) in a study on 129,000 pregnant women that was recently reviewed (Elsaddig \& Khalil, 2021). In another study, $21 \%$ of the newborns had respiratory distress syndrome (Mark et al, 2021). A metaanalysis of 19 studies suggested that $57 \%$ of the neonates of infected mothers have been admitted to neonatal intensive care nurseries, although this review included only 41 COVID-infected mothers (DiMascio et al, 2020).

Elevated prematurity rates have been reported in virtually all of the reviews in this literature. Although it is not clear whether COVID-19 is an independent risk factor for prematurity, there is consensus that the rates are higher for pregnant women with COVID (Wastnedger et al, 2021). As already noted, some have referred to prematurity as iatrogenic, following elective Caesarean sections indicated by the symptoms of the infected women. (Moore \& Suthar, 2021; Wastnedger et al, 2021). Preterm birth in these neonates has also been associated with necrotizing enterocolitis (inflammatory disease of the G.I. tract) and bronchopulmonary dysplasia (Prochaska et al, 2020).

Low birthweight has also been reported for the offspring of COVIDinfected pregnant women (Elsaddig \& Khalil, 2021; Wastnedger et al, 2021). The greater prevalence of low birthweight noted in these reviews was based on very large samples.

The literature has been mixed on Apgar scores. In one large sample survey, no differences were noted between the offspring of women with COVID versus those without COVID (Elsaddig \& Khalil, 2021).But in another review, Apgar scores were lower in the neonates of women with COVID (Kumar et al, 2021). Despite these problems, the authors of one of these reviews suggested that there may have been "less newborn problems than expected due to travel bans and greater emphasis on infection protection" (Elsaddig \& Khalil, 2021).

Although the data are mixed on newborn infection, some have expressed concern that maternal inflammation is a "Neurodevelopmental disease primer"(Prochaska et al, 2020). Examples these authors have given for the long-term effects on children of mothers with inflammatory diseases included autism spectrum disorder, depression and bipolar disorder (Prochaska et al, 2020). And elevated C-reactive protein has also lead to neuropsychological disorders in children (Abdali et al, 2020). Although developmental follow-ups of children of COVID infected mothers have not yet appeared in the literature, the inflammatory 
condition of the COVID infected pregnant women and the potential vertical transmission could ostensibly lead to these developmental disorders

\section{Vaccine Effects on Pregnancy}

Although at least one lengthy review has been published in 2020 on the importance of vaccines and the development of specific vaccines for pregnant women, no mention was made about COVID-19 vaccines (Maertens et al, 2020). As in this review, the authors of another recent review have reiterated that the pregnant woman's immunity is thought to pass through the placenta (Kumar et al, 2020). Specifically, IgG antibodies have actively been transferred through the placenta to provide passive immunity for the newborn (Kumar et al, 2020). The transfer of IgG antibodies are uniquely thought to cross the placenta as, for example, IgA antibodies reputedly cannot cross the placenta, as already mentioned. Although pregnant women have been cited as a priority for vaccination, no studies could be found in the COVID literature on the effects of vaccine on pregnant women.

At least one research group has recommended the inclusion of pregnant women in vaccine trials. As the authors said, "First, these vaccines contain mRNA encapsulated in a lipid nanoparticle that is delivered into host cells. The body's own host cells generate coronavirus spike proteins that stimulate antibodies against SARS-CoV-2. This activity occurs robustly in regional lymph nodes. There is no biological reason to suspect that this process is different during pregnancy, so we expect similar efficacy in pregnant and non-pregnant persons. Second, these vaccines contain no live virus or adjuvants that could affect the developing fetus. Further, available data about developmental and reproductive toxicity generated from rats administered the Moderna mRNA vaccine show no safety signals concerning female reproduction, fetal or embryonal development, or postnatal development "(Riley \& Jamieson, 2021). In light of these recommendations, it is noteworthy that data could not be found on the effects of current vaccines on pregnant women and their fetuses.

\section{Reproductive Health, Fertility and Vaccines During COVID}

\section{Reproductive Health}

COVID-19 has had a significant impact on women's reproductive health as reported in a recent survey on 1031 women (with a mean age of 37) (Phelan et al, 2021). In this survey, $46 \%$ of women reported a change in their menstrual cycle during COVID-19, 53\% worsening premenstrual symptoms, $18 \%$ menorrhagia (heavy bleeding), $30 \%$ new dysmenorrhea (cramps and pelvic pain with menstruation) and $73 \%$ missed periods. Although there was a reported increase in weight, there was also a 30minute increase in weekly exercise. $50 \%$ of the women said their diet was worse and $40 \%$ reported working more. They also self-reported a significant increase in depressed mood, poor appetite, binge eating, poor concentration, anxiety, poor sleep, loneliness and excess alcohol use. More specific stressors included $48 \%$ work stress and $25 \%$ having difficulty accessing healthcare. Lower prevalence data were noted for financial difficulties, homeschooling or childcare, family and partner conflict and family illness.

These data suggest that even without infection, women during COVID-19 have experienced negative effects on their reproductive health. The anxiety surrounding potential infection and/or the physiological/biochemical effects of lockdown, social distancing and limited activity may have contributed to these effects.

\section{Fertility}

Although women's fertility would seemingly also be affected by COVID-19, the only literature has focused on women's stress surrounding the closing of fertility clinics during the pandemic (BenKimhy et al, 2020). Many studies, in contrast, have explored the specific effects of COVID-19 on male fertility. A recent review on COVID-19 effects on male fertility, for example, concluded that although there are limited data, viral mRNA has been identified in the semen of infected men, with some evidence of altered seminal parameters (Khalili et al, 2020). Low testosterone and dihydrotestosterone with increased luteinizing hormone were also reported for pregnant women with infection in this review paper.

\section{Vaccine Effects on Fertility}

Only two studies could be found in the literature on vaccine effects on women's fertility. In the first study, the full human dose of the Pfizer vaccine was given intramuscularly to 44 female rats on the 21 st and 14th day prior to mating and then on gestation days nine and 20 (the end of lactation)(Bowman et al, 2021). No effects were noted on the mating performance, the fertility, the embryo, fetus or neonate. In addition, postnatal development was normal including growth, physical development and neurological functions at the end of lactation. The authors concluded that the Pfizer vaccine was safe for women's fertility, pregnancy and offspring.

Similarly, the Pfizer vaccine had no negative effects on human women's fertility in a study conducted in Israel (Safrai et al 2021). Only intracytoplasmic sperm injection (ICSI) patients were included in the study ( $\mathrm{N}=47)$. As has been noted, this in vitro fertilization procedure involves injection of a single sperm cell directly into the cytoplasm of an egg in cases where the egg's outer layer may be difficult to penetrate or the sperm unable to swim. As the authors reported, the characteristics of the ICSI cycles were similar before and after the vaccinations and the number of clinical pregnancies did not differ between the pre-and postvaccination groups. The authors concluded that the Pfizer vaccine appeared to have no impact on the women's fertility.

\section{Methodological Limitations of this Literature}

Many methodological limitations of this literature can be mentioned including that most of the publications are reviews of prevalence studies that are based on data from medical records. As such, the studies have been focused on infection prevalence and laboratory measures of maternal infection, antibodies in placental and umbilical tissues and positivity of neonatal oropharyngeal swabs, all of which are critically informative for clinical treatment. However, there are many potentially confounding variables that have not been assessed in this research.

Very few of the studies, for example, have entered the potentially confounding demographic variables of ethnicity, socioeconomic status, maternal age and parity in their analyses even though these variables have notably affected pregnancies and neonatal outcomes in previous studies and could readily be derived from medical records. Further, behavioral variables that may not have appeared in most medical records such as the consumption of alcohol, illicit drugs, tobacco, caffeine and diet as well as weight gain and sedentary behavior have had notable effects in preCOVID studies but have not been considered as potentially compounding the effects of infection on COVID-19 pregnancies.

As other examples of confounding variables, mood states and sleep disturbances are not only emotional but also have physiological and biochemical effects. Pregnant women are notably worried/fearful of infection effects not only on themselves but on their fetus and newborn (Elsaddig \& Khalil, 2021; Mirbeyk et al, 2021; Wastnedger et al, 2021). Infection worries can lead to anxiety and depression even in women without those pre-existing mood state problems (Field et al, 2021). PreCOVID data have documented the comorbidity of depression, anxiety and anger in pregnant women and the accompanying physiological and biochemical effects (Field et al, 2003). These have included low vagal 
activity, right frontal EEG activation, increased cortisol and norepinephrine levels and lower dopamine and serotonin levels. These variables, in turn, have significantly predicted prematurity and low birthweight in pre-COVID structural equation modeling analyses (Field et al, 2006). The degrees to which these effects may be contributing to prenatal and postpartum effects of COVID infection have not been assessed even though hundreds of studies have documented COVID-19 associated worries, anxiety, depression and sleep disturbances in noninfected folks during the pandemic (Field et al, 2021). All of these measures could be readily taken from brief self-report scales, Fitbit watches and from saliva samples in future studies.

The data have also been cross-sectional and derived from typically small samples of symptomatic, hospitalized pregnant women at different gestational ages and different times during the pandemic. This variability in the time frames of the data collection and in the variability in the severity of the infection effects at variable gestational ages would contribute to the mixed findings in this literature. The cross-sectional data could not be interpreted for causality or direction of effects. Longitudinal, multivariate studies are needed that control for these variables and those just listed.

Aside from the Apgar test that is routinely taken at birth and reported in two conflicting studies in this literature (Elsaddig \& Khalil, 2021; Kumar et al, 2021), there are no other neonatal assessments that have appeared in this literature. For example, the Brazelton Neonatal Behavior Assessment Scale that includes neurological and behavioral items and that has been routinely administered in most neonatal intensive care nurseries in recent years has not been given in the studies on neonatal outcomes. That is surprising inasmuch as many assessments of that kind had been made decades ago on HIV-exposed, Cocaine-exposed and even caffeineexposed neonates. However, neonatal neurological and behavioral data are not routinely entered into medical records. Although some have speculated that COVID-infection could have serious neurological and behavioral effects in later childhood, there are virtually no data on those effects on the neonate. Neurological signs during the neonatal period have been significant predictors of later neurological dysfunction and importantly can help differentiate prenatal from postnatal environmental effects.

Despite the relative longevity of the COVID-19 pandemic and the negative effects reported on infected pregnancies early in the pandemic, no intervention studies other than medical interventions could be found in this literature. At least educational and physical activity zoom sessions could be offered to pregnant women who are infected or those at risk for infection or even those at risk for negative effects due to worries and anxiety/depression/sleep problems during COVID. And, hotlines and supportive services in addition to the intensive care that has been critically provided could invariably help infected pregnant women, their fetuses and their newborns during this COVID-19 pandemic. Research on these, in turn, would help inform future efforts to reduce the psychological and physical effects associated with COVID-19 in pregnant women.

\section{References}

1. Abdoli A, Falahi S, Kenarkoohi A, Shams M, Mir H, Jahromi MAM. (2020). The COVID-19 pandemic, psychological stress during pregnancy, and risk of neurodevelopmental disorders in offspring: a neglected consequence. J Psychosom Obstet Gynaecol 41: 247-248,

2. Akgor U, Fadıloglu E, Soyak B, Unal C, Cagan M, Temiz BE, Erzenoglu BE, Ak S, Gultekin M, Ozyuncu O. Anxiety, depression and concerns of pregnant women during the COVID-19 pandemic. Arch Gynecol Obstet. 2021 Jul; 304 (1):125-130. doi: 10.1007/s00404-020-05944-1. Epub 2021 Jan 12. PMID: 33433702; PMCID: PMC7802427.
3. Alberca RW, Pereira NZ, Oliveira LMDS, Gozzi-Silva SC and Sato MN (2020) Pregnancy, Viral Infection, and COVID-19. Front. Immunol. 11:1672. doi: 10.3389/fimmu.2020.01672

4. Ashraf MA, Keshavarz P, Hosseinpour P, Erfani A, Roshanshad A, Pourdast A, Nowrouzi-Sohrabi P, Chaichian S, Poordast T. (2020). Coronavirus Disease (COVID-19): A Systematic Review of Pregnancy and the Possibility of Vertical Transmission. J Reprod Infertil. 21(3):157-168. PMID: 32685412; PMCID: PMC7362089.

5. Ben-Kimhy R, Youngster M, Medina-Artom TR, Avraham S, Gat I, Marom Haham L, Hourvitz A, Kedem A. (2020). Fertility patients under COVID-19: attitudes, perceptions and psychological reactions. Hum Reprod. Dec 1; 35(12):27742783. doi: 10.1093/humrep/deaa248. PMID: 32877507; PMCID: PMC7499650.

6. Bowman, C. Bouressam, M. Campion, S. Cappon, G. Catlin, N. Cutler, M. (2021). Lack of effects ofemale fertility and prenatal and postnatal offspring development in rats with BNT162b2,mRNA-based COVID-19 vaccine 'Reproductive Toxicology, 103, 28-35

7. Chi J, Gong W, Gao Q. (2021). Clinical characteristics and outcomes of pregnant women with COVID-19 and the risk of vertical transmission: a systematic review. Arch Gynecol Obstet. 303(2):337-345. doi: 10.1007/s00404-020-05889-5. Epub 2020 Dec 1. PMID: 33258995; PMCID: PMC7706177.

8. Di Mascio D, Khalil A, Saccone G, Rizzo G, Buca D, Liberati M, Vecchiet J, Nappi L, Scambia G, Berghella V, D'Antonio F. (2020). Outcome of coronavirus spectrum infections (SARS, MERS, COVID-19) during pregnancy: a systematic review and meta-analysis. Am J Obstet Gynecol MFM. 2020 May; 2(2):100107. doi: 10.1016/j.ajogmf.2020.100107. Epub. PMID: 32292902; PMCID: PMC7104131.

9. Elsaddig M, Khalil A. (2021). Effects of the COVID pandemic on pregnancy outcomes. Best Pract Res Clin Obstet Gynaecol. 2021 Jun 73:125-136. doi: 10.1016/j.bpobgyn.2021.03.004. Epub. PMID: 33832868; PMCID: PMC7969862.

10. Fan S, Guan J, Cao L, Wang M, Zhao H, Chen L, Yan L. (2020).Psychological effects caused by COVID-19 pandemic on pregnant women: A systematic review with meta-analysis. Asian J Psychiatr. 2021 Feb; 56:102533. doi: 10.1016/j.ajp.2020.102533. Epub. PMID: 33418283; PMCID: PMC7833174.

11. Field, T., Diego, M., Hernandez-Reif, M., Schanberg, S., Kuhn, C., Yando, R. \& Bendell, D. (2003). Pregnancy Anxiety and Comorbid Depression and Anger Effects on the Fetus and Neonate. Depression and Anxiety, 17, 140-151.

12. Field, T., Hernandez-Reif, M., Diego, M., Figueiredo, B., Schanberg, S., \& Kuhn, C. (2006). Prenatal Cortisol, Prematurity and Low Birthweight. Infant Behavior and Development, 29, 268-275.

13. Field, T. Mines, S., Poling, S., Bendell, D. \& Veazey, C. (2021). Stressors and buffers during a COVID-19 lockdown: A narrative review. American Journal of Psychiatric Research and Reviews. 3, 1, 1-18.

14. Glover V. (2015). Prenatal stress and its effects on the fetus and the child: possible underlying biological mechanisms. Adv Neurobiol. 10:269-83. doi: 10.1007/978-1-4939-1372-5_13. PMID: 25287545.

15. Hocaoglu M, Ayaz R, Gunay T, Akin E, Turgut A, Karateke A. (2020). Anxiety and Post-Traumatic Stress Disorder Symptoms in Pregnant Women during the COVID-19 Pandemic's Delay Phase. Psychiatr Danub. Autumn-Winter;32(3-4):521-526. doi: 10.24869/psyd.2020.521. PMID: 33370762. 
16. Khalili MA, Leisegang K, Majzoub A, Finelli R, Panner Selvam MK, Henkel R, Mojgan M, Agarwal A. (2020). Male Fertility and the COVID-19 Pandemic: Systematic Review of the Literature. World J Mens Health. 38(4):506-520. doi: 10.5534/wjmh.200134. Epub 2020 Aug 14. PMID: 32814369; PMCID: PMC7502312.

17. Kumar R, Yeni CM, Utami NA, Masand R, Asrani RK, Patel SK, Kumar A, Yatoo MI, Tiwari R, Natesan S, Vora KS, Nainu F, Bilal M, Dhawan M, Emran TB, Ahmad T, Harapan H, Dhama K. (2021). SARS-CoV-2 infection during pregnancy and pregnancy-related conditions: Concerns, challenges, management and mitigation strategies-a narrative review. J Infect Public Health. 14(7):863-875. doi: 10.1016/j.jiph.2021.04.005. Epub 2021 Apr 23. PMID: 34118736; PMCID: PMC8062420.

18. Maertens K, Orije MRP, Van Damme P, Leuridan E. (2020). Vaccination during pregnancy: current and possible future recommendations. Eur J Pediatr. 2020 Feb; 179(2):235-242. doi: 10.1007/s00431-019-03563-w. Epub. PMID: 31912233; PMCID: PMC7222942.

19. Maleki Dana P, Kolahdooz F, Sadoughi F, Moazzami B, Chaichian S, Asemi Z. (2020). COVID-19 and pregnancy: a review of current knowledge. Infez Med.; 28(suppl 1):46-51. PMID: 32532938.

20. Mark EG, McAleese S, Golden WC, Gilmore MM, SickSamuels A, Curless MS, Nogee LM, Milstone AM, Johnson J. (2021). Coronavirus Disease 2019 in Pregnancy and Outcomes Among Pregnant Women and Neonates: A Literature Review. Pediatr Infect Dis J. May 1; 40(5):473-478. doi: 10.1097/INF.0000000000003102. PMID: 33847297; PMCID: PMC8048372.

21. Mirbeyk M, Saghazadeh A, Rezaei N. (2021).A systematic review of pregnant women with COVID-19 and their neonates. Arch Gynecol Obstet. 2021 Jul; 304(1):5-38. doi: 10.1007/s00404-021-06049-z. Epub. PMID: 33797605; PMCID: PMC8017514.

22. Moore KM, Suthar MS. (2021). Comprehensive analysis of COVID-19 during pregnancy. Biochem Biophys Res Commun.; 538:180-186. doi: 10.1016/j.bbrc.2020.12.064. Epub 2020 Dec 24. PMID: 33384142; PMCID: PMC7759124

23. Moyer CA, Compton SD, Kaselitz E, Muzik M. (2020). Pregnancy-related anxiety during COVID-19: a nationwide survey of 2740 pregnant women. Arch Womens Ment Health. 2020 Dec;23(6):757-765. doi: 10.1007/s00737-020-01073-5. Epub 2020 Sep 29. PMID: 32989598; PMCID: PMC7522009.

24. Narang K, Enninga EAL, Gunaratne MDSK, Ibirogba ER, Trad ATA, Elrefaei A, Theiler RN, Ruano R, Szymanski LM, Chakraborty R, Garovic VD. (2020). SARS-CoV-2 Infection and COVID-19 During Pregnancy: A Multidisciplinary Review. Mayo Clin Proc. 2020 Aug; 95(8):1750-1765. doi: 10.1016/j.mayocp.2020.05.011. Epub 2020 May 30. PMID: 32753148; PMCID: PMC7260486.

25. Panahi L, Amiri M, Pouy S. (2020). Risks of Novel Coronavirus Disease (COVID-19) in Pregnancy; a Narrative Review. Arch Acad Emerg Med.; 8(1):e34. PMID: 32232217; PMCID: PMC7092922.

26. Pettirosso E, Giles M, Cole S, Rees M. (2020). COVID-19 and pregnancy: A review of clinical characteristics, obstetric outcomes and vertical transmission. Aust $\mathrm{N} \mathrm{Z} \mathrm{J} \mathrm{Obstet}$
Gynaecol. 2020 Oct; 60(5):640-659. doi: 10.1111/ajo.13204. Epub. PMID: 32779193; PMCID: PMC7436616.

27. Phelan N, Behan LA, Owens L. (2021). The Impact of the COVID-19 Pandemic on Women's Reproductive Health. Front Endocrinol (Lausanne). 2021 Mar 22; 12:642755. doi: 10.3389/fendo.2021.642755. PMID: 33841334; PMCID: PMC8030584.

28. Prochaska E, Jang M, Burd I. (2020).COVID-19 in pregnancy: Placental and neonatal involvement. Am J Reprod Immunol; 84(5):e13306. doi: 10.1111/aji.13306. Epub 2020 Aug 15. PMID: 32779810; PMCID: PMC7404599.

29. Ravaldi C, Ricca V, Wilson A, Homer C, Vannacci A. (2020). Previous psychopathology predicted severe COVID-19 concern, anxiety, and PTSD symptoms in pregnant women during "lockdown" in Italy. Arch Womens Ment Health. Dec;23(6):783-786. doi: 10.1007/s00737-020-01086-0. Epub Nov 20. PMID: 33215247; PMCID: PMC7677012.

30. Riley, L. \& Jamieson, D. (2021). Inclusion of Pregnant and Lactating Persons in COVID-19 Vaccination Efforts. Ann Intern Med.2021; 174:701-702. [Epub ahead of print 26 January 2021]. doi:10.7326/M21-0173

31. Ryan GA, Purandare NC, McAuliffe FM, Hod M, Purandare CN. (2020). Clinical update on COVID-19 in pregnancy: A review article. J Obstet Gynaecol Res. 2020 Aug; 46(8):12351245. doi: 10.1111/jog.14321. Epub 2020 Jun 4. PMID: 32500549; PMCID: PMC7300676.

32. Safrai, M. Rottenstreich, A. Herzberg, S. Imbar, T. Reubinoff, B. Ben-Meir, A. (2021). Stopping the misinformation: BNT162b2 COVID-19 vaccine has no negative effect on women's fertility, 2021, online ahead of print.

33. Schwartz DA.(2021). An Analysis of 38 Pregnant Women With COVID-19, Their Newborn Infants, and Maternal-Fetal Transmission of SARS-CoV-2: Maternal Coronavirus Infections and Pregnancy Outcomes. Arch Pathol Lab Med. 2020 Jul 1; 144(7):799-805. doi: 10.5858/arpa.2020-0901-SA. PMID: 32180426.

34. Sun F, Zhu J, Tao H, Ma Y, Jin W. A (2021). systematic review involving 11,187 participants evaluating the impact of COVID19 on anxiety and depression in pregnant women. J Psychosom Obstet Gynaecol. Jun; 42(2):91-99. doi: 10.1080/0167482X.2020.1857360. Epub 2020 Dec 17. PMID: 33327827.

35. Tomfohr-Madsen LM, Racine N, Giesbrecht GF, Lebel C, Madigan S. (2021). Depression and anxiety in pregnancy during COVID-19: A rapid review and meta-analysis. Psychiatry Res. 300:113912. doi: 10.1016/j.psychres.2021.113912. Epub Apr 1. PMID: 33836471 .

36. Wang Y, Chen L, Wu T, Shi H, Li Q, Jiang H, Zheng D, Wang X, Wei Y, Zhao Y, Qiao J. (2020). Impact of Covid-19 in pregnancy on mother's psychological status and infant's neurobehavioral development: a longitudinal cohort study in China. BMC Med. 18(1):347. doi: 10.1186/s12916-02001825-1. PMID: 33143711; PMCID: PMC7609382.

37. Wastnedge EAN, Reynolds RM, van Boeckel SR, Stock SJ, Denison FC, Maybin JA, Critchley HOD. (2020). Pregnancy and COVID-19. Physiol Rev. 2021 Jan 1; 101(1):303-318. doi: 10.1152/physrev.00024.. Epub. PMID: 32969772; PMCID: PMC7686875. 